\title{
The Neuroanatomy of Reproduction: Seeing Is Believing
}

\author{
Alice S. Rhoton-Vlasak, MD ${ }^{4}$ \\ ${ }^{1}$ College of Medicine, University of Florida, Gainesville, Florida \\ ${ }^{2}$ Department of Obstetrics and Gynecology, University of Florida, \\ Gainesville, Florida \\ 3 Department of Neurosurgery, University of Florida, Gainesville, Florida \\ ${ }^{4}$ Division of Reproductive Endocrinology and Infertility, Department of \\ Obstetrics and Gynecology, University of Florida, Gainesville, Florida
}

Alexander L. Vlasak, BS ${ }^{1}$ Amelia Schaub, MD² Margaret E. "Robin" Barry, MA ${ }^{3}$

\begin{abstract}
Address for correspondence Alice S. Rhoton-Vlasak, MD, Division of Reproductive Endocrinology and Infertility, Department of Obstetrics and Gynecology, University of Florida, PO Box 100294, Gainesville, FL 32610 (e-mail: Rhotona@ufl.edu).
\end{abstract}

Semin Reprod Med 2019;37:251-256

\begin{abstract}
Keywords

- pituitary

- reproduction

- hypothalamicpituitary axis

- neuroanatomy

The brain is the central controller of reproduction and the menstrual cycle. Reproductive endocrinologists spend their days treating patients with perturbations in reproduction as a result of pituitary diseases and manipulate pituitary hormones to enhance fertility and quality of life. Microscopic neuroanatomical images will allow a better understanding of how a tumor in the pituitary might affect vision, or a mass in the brain might cause amenorrhea. Clinical correlations that are taught every day become much clearer once the anatomical relationships are explored. The objective of this pictorial tour is to elucidate anatomical and clinical relationships while showcasing the neuroanatomy of reproduction.
\end{abstract}

The pituitary gland controls important body functions and the hormonal system. It is a protrusion at the base of the brain and similar in size to a pea, which is why it is also called "hypophysis" (which is Greek for "growth attached below"). It sits in the sella turcica of the sphenoid bone, immediately behind and superior to the sphenoid sinus. The pituitary gland is sometimes called the "master endocrine gland" because it secretes hormones that directly affect the way many parts of the body work. It also modulates secretion of hormones made by many other glands in the body and ultimately contributes to the control of the involuntary nervous system, which manages the balance of energy, heat, and water in the body. It is through the hormonal action of the pituitary that the brain acts as the central modulator of reproductive function and the menstrual cycle. Reproductive endocrinologists frequently care for patients with imbalances in these hormones, in conditions such as hyperprolactinemia, anovulation, and hypothalamic amenorrhea. It is through the pituitary that the brain acts as the central controller of reproduction and the menstrual cycle. In the early 20th century, researchers such as Crowe and Aschner made several pioneering discoveries demonstrating the connection of the pituitary gland to gonadal function. ${ }^{1}$ Over the next 40 years, gonadotropin-releasing hormone $(\mathrm{GnRH})$, follicle-stimulating hormone (FSH), and luteinizing hormone (LH) were discovered; eventually purified; and are now mass produced for the treatment of a variety of endocrine disorders. Reproductive endocrinologists frequently care for patients with disturbances in reproduction as a result of pituitary pathology and manipulate the balance of pituitary hormones to restore or enhance fertility to patients.

Schioffer of Vienna successfully performed the first transsphenoidal pituitary tumor resection in 1907. Surgical approaches to the pituitary were later refined by the likes of Harvey Cushing and other neurosurgeons and eventually made increasingly safe, accurate, and less invasive through technological advancements such as the surgical microscope, endoscope, and eventually MRI and CT-based surgical guidance. Of note is the fact that nearly half a century ago, it would be a rarity to see a patient's pituitary function preserved following the removal of a pituitary tumor. Not only was increased treatment success achieved through the technological developments described earlier, it was also achieved through the intricate study of the skull base, cranial nerves, and pituitary structure and blood supply by dedicated neurosurgeons throughout the world. Even drugs, such as the dopamine agonists, bromocriptine and cabergoline,
Issue Theme Reproductive Health Needs of the Military and Veterans: Part 3; Guest Editors, Alicia Y. Christy, MD, MHSCR, FACOG and Gilbert L. Mottla, MD
Copyright @ 2019 by Thieme Medical Publishers, Inc., 333 Seventh Avenue, New York, NY 10001, USA.

Tel: +1(212) 760-0888.
DOI https://doi.org/ 10.1055/s-0038-1675585. ISSN 1526-8004. 
used to treat hyperprolactinemia and prolactinomas, can be better understood in the context of neural connections. In much the same way as the application of microsurgery, learned through a better understanding of microanatomy, has improved surgical outcomes, it is our belief that solidifying the links between microanatomy and reproductive endocrinology will lead to improved understanding of normal reproductive processes in addition to improved clinical recognition and treatment of perturbations of reproduction due to pituitary and intracranial diseases.

The motivation for this article comes from the work of Dr. Albert Rhoton Jr., a neurosurgeon who dedicated his career to the study of neuroanatomy. The extensive brain mapping and anatomical dissections performed in his laboratory were aimed at improving the quality of care for neurosurgical patients and have been used to educate physicians around the world. The images seen in this article are the products of the dedication of Dr. Rhoton, his international laboratory fellows, and the medical illustrators he worked with.

\section{The Pituitary and Reproduction}

The critical parts of the female reproductive system are the hypothalamus, pituitary, and female genital tract, with the ovaries linking the brain and reproduction. These anatomical components and their actions form the basis of the hypothalamic-pituitary-gonadal axis. Any disease process that interrupts hypothalamic or pituitary function and disturbs the homeostasis of this physiologic axis will alter normal reproductive events. The endocrine feedback that leads to ovulation is orchestrated by the ovarian hormone feedback on the hypothalamus and anterior pituitary. For the reproductive endocrinologists, these structures require careful consideration as sources of pathology and often serve as the target of treatments used to enhance fertility. In addition, most forms of ovulation induction and ovarian stimulation utilize hormones and feedback loops based on our understanding of the pituitary-ovarian axis.

\section{Pituitary and Hypothalamic Anatomy}

The pituitary sits at the base of the brain in the sella turcica of the sphenoid bone. It can be anatomically divided into two distinct lobes, anterior and posterior. The posterior lobe, or neuro-hypophysis, is multinodular and lighter in color than the anterior lobe. The anterior lobe, or adeno-hypophysis, is larger and wraps partially around the infundibular stalk to form an incomplete collar called the pars tuberalis ${ }^{2}$ (-Figs. 1-3). Immediately superior to the pituitary sit the optic nerves and optic chiasm. Laterally, the cavernous segments of the internal carotid arteries flank the pea-sized gland (-Fig. 4). Posterior to the optic chiasm, the infundibular stalk connects the pituitary and the hypothalamus. The anatomical relationship between pituitary and hypothalamus is further illustrated from a sagittal view (-Fig. 5). The stalk is composed of two distinct sections: a vascular path and a neuronal path, which correlate with the anterior and posterior pituitary, respectively. ${ }^{3}$

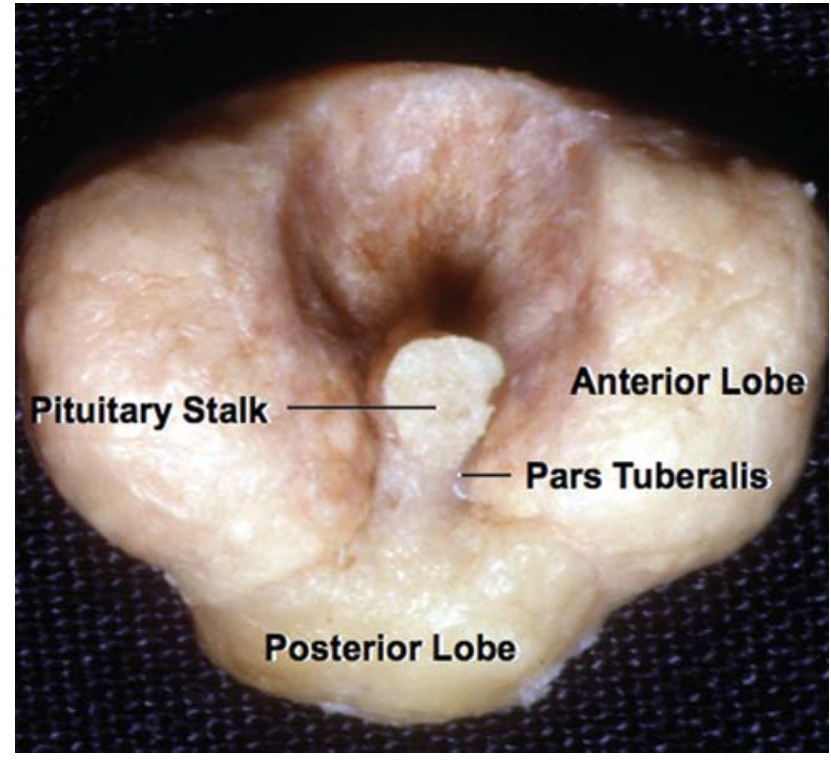

Fig. 1 The superior surface of the pituitary gland. (Reprinted with permission from Rhoton AL. The stellar region. Neurosurgery 2002;53:336.)

\section{Pituitary Stalk and Hormones}

The posterior path, or hypothalamic neuro-hypophyseal tract, contains neurons originating in the hypothalamus and coursing down onto the anterior surface of posterior pituitary (-Fig. 6). The hormones of the posterior system (antidiuretic hormone $[\mathrm{ADH}]$ and oxytocin) are produced in the cell bodies of the neurons, located in the hypothalamus. Axons of these cells run down the stalk and ultimately release ADH and oxytocin into the extracellular space whereby they pass directly into the posterior pituitary. ADH and oxytocin can be stored in the posterior pituitary for later release. The

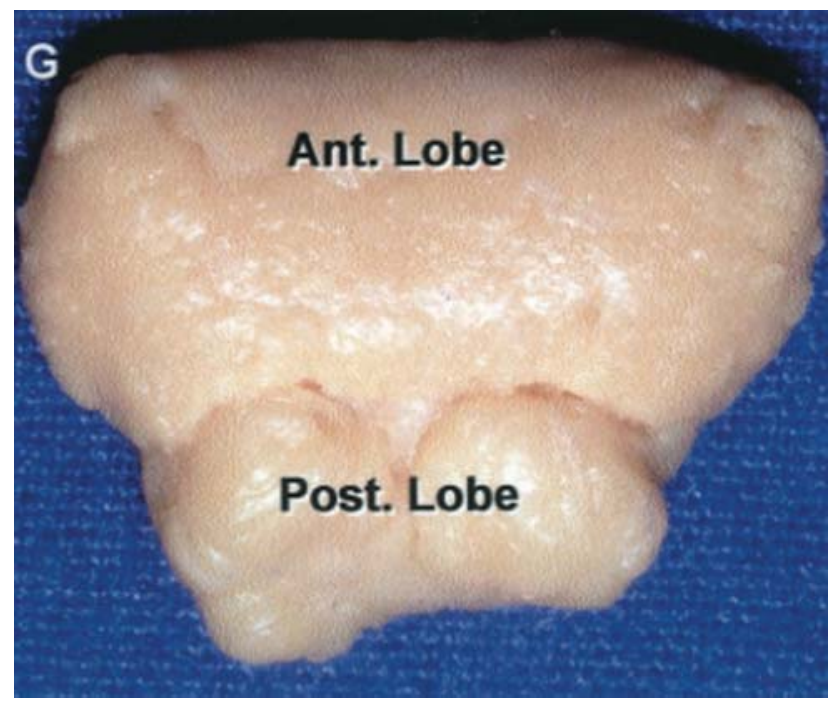

Fig. 2 The inferior surface of the pituitary. (Reprinted with permission from Rhoton AL. The stellar region. Neurosurgery 2002;53:336.) 


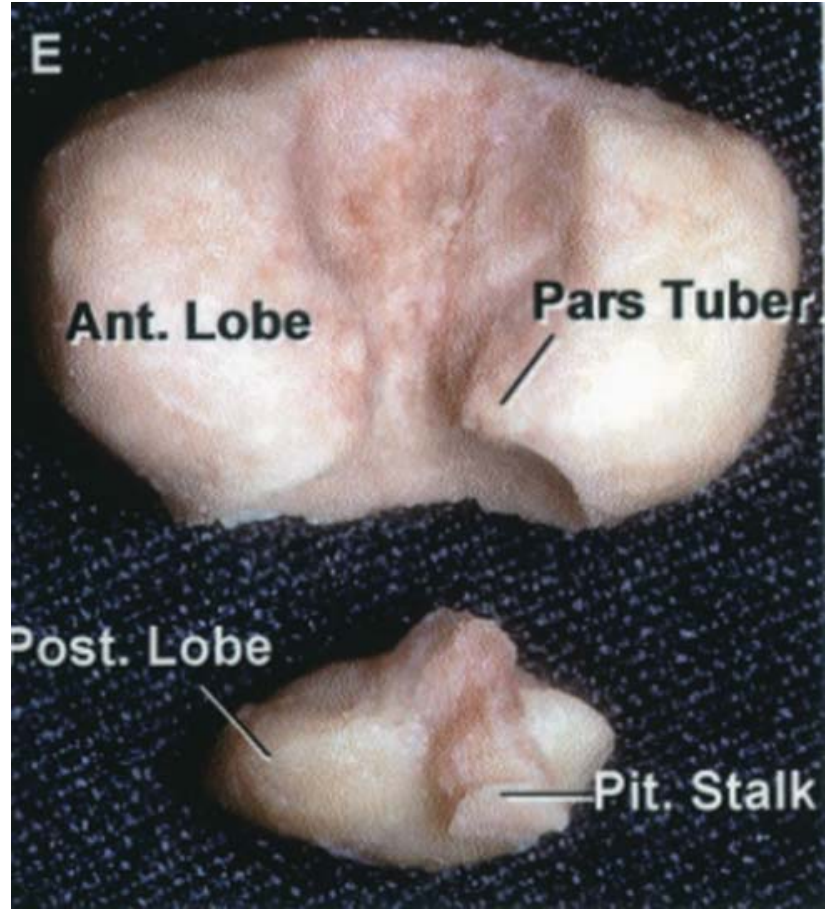

Fig. 3 The anterior and posterior portions of the pituitary separated. (Reprinted with permission from Rhoton AL. The stellar region. Neurosurgery 2002;53:336.)

anterior stalk composes a portion of the hypothalamic-hypophyseal portal system, which allows hormones synthesized in the hypothalamus (i.e., growth hormone [GH]-releasing hormone) to diffuse through a vascular network to their targets in the anterior pituitary (-Fig. 6). This results in the stimulation or inhibition of the release of hormones synthesized in the anterior pituitary (FSH, LH, thyroid-stimulating hormone [TSH], adrenocorticotropic hormone [ACTH], prolactin, and $\mathrm{GH}$ ) which are ultimately released into the bloodstream to act on their target organs ( - Fig. 7 ).

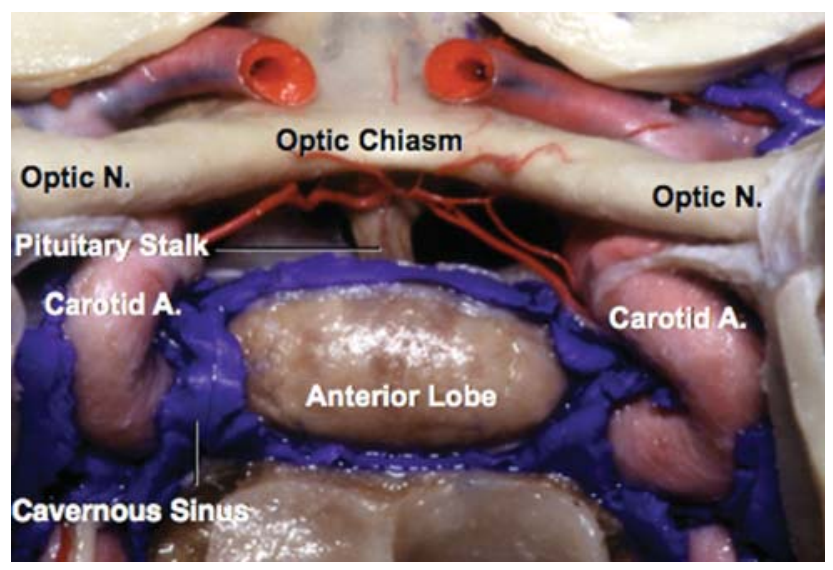

Fig. 4 An anterior coronal view of the pituitary. The gland is below the optic nerves and chiasm, and between the cavernous segments of the internal carotid artery. (Photo courtesy of the Rhoton Collection.)

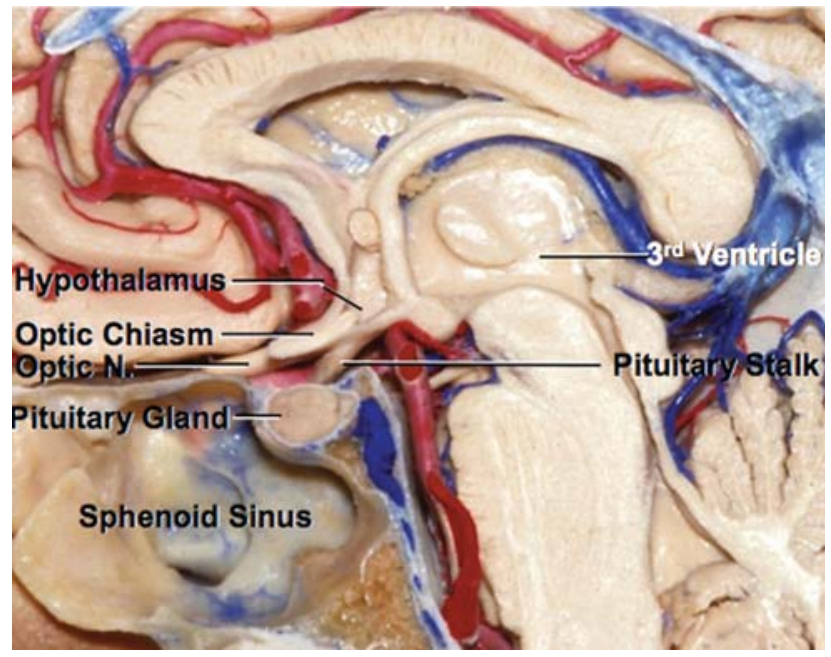

Fig. 5 Mid sagittal section of the pituitary region. (Photo courtesy of the Rhoton Collection).

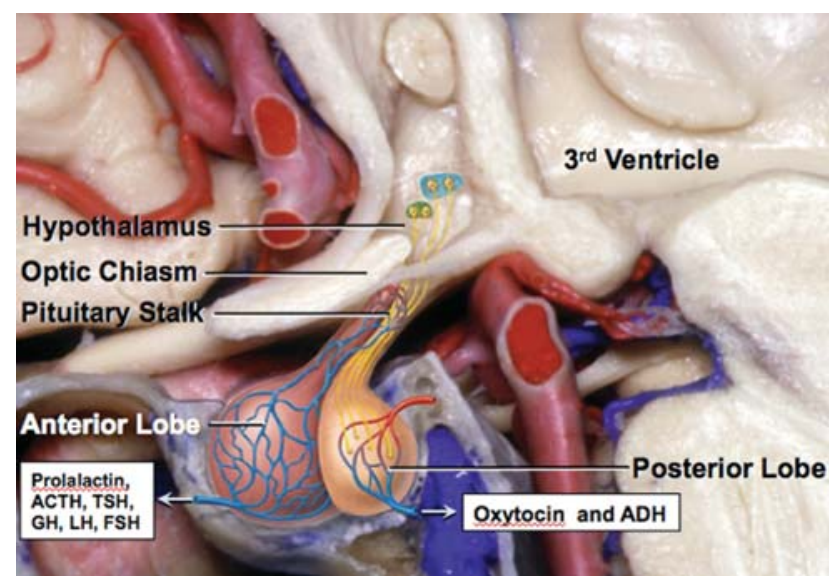

Fig. 6 Depiction of the neuronal and vascular paths connecting the hypothalamus and the pituitary.

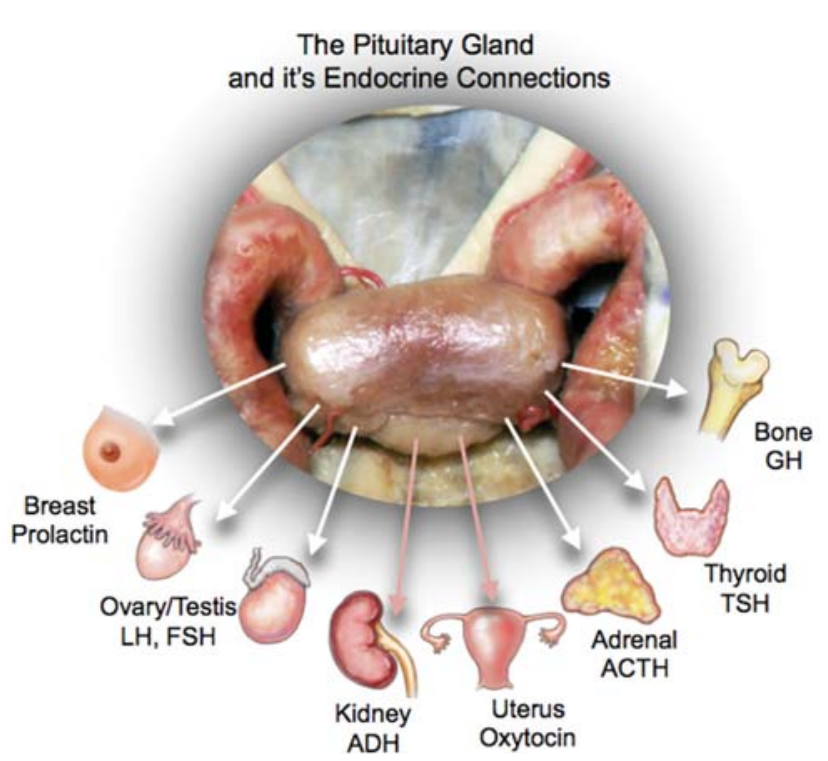

Fig. 7 Hormones released by the anterior and posterior pituitary and their target organs. 


\section{Pituitary Tumors}

Pituitary adenomas or tumors account for approximately $15 \%$ of all intracranial tumors ${ }^{4-6}$ and the population prevalence of pituitary tumors has been estimated between 10 and 20\%. ${ }^{7-10}$ They can be categorized by their adeno-hypophysial cell linages, and when hormonally active can lead to a variety of endocrine consequences. Lactotroph adenomas (also known as prolactinomas) or prolactin-releasing tumors are the most common hormone producing tumors leading to amenorrhea, galactorrhea, loss of libido, osteoporosis, infertility, and erectile dysfunction in males. ${ }^{11-13} \mathrm{GH}$-releasing tumors (somatotroph adenomas) result in acromegaly, a serious systemic condition caused in more than $98 \%$ of cases by a pituitary tumor secreting excessive $\mathrm{GH} .{ }^{14}$ These tumors can lead to a variety of systemic complications including diabetes mellitus, hypertension, arthritis, carpal tunnel syndrome, and sleep apnea. ${ }^{11,13}$ In 30 to $40 \%$ of women with $\mathrm{GH}$-secreting pituitary tumors, hyperprolactinemia with or without galactorrhea is also present and roughly the same percentage will experience amenorrhea or infertility. ${ }^{15}$ ACTH-releasing tumors (corticotroph adenomas) cause Cushing's disease and in approximately $75 \%$ of female patients, gonadal dysfunction is present, as well as hirsutism and alopecia. ${ }^{11,13}$ TSH-releasing tumors (thyrotroph adenomas) account for only 0.5 to $2 \%$ of all pituitary adenomas but typically present with symptoms of hyperthyroidism. ${ }^{13,16}$ Gonadotroph adenomas typically are nonfunctioning, and do not secrete any hormones. In rare cases when they release FSH or $\mathrm{LH}$, they can cause polycystic ovarian syndrome, menstrual irregularities, ovarian hyperstimulation syndrome, sexual dysfunction, and testicular hypertrophy in males. ${ }^{11,17}$ Common locations of various functional tumors are shown in - Fig. 8. Remaining tumor types include null cell adenoma, plurihormonal adenoma, pituitary carcinoma, and pituitary blastoma. ${ }^{13}$

Pituitary tumors can also be categorized by radiologic size. Those measuring greater than $1 \mathrm{~cm}$ are macroadenomas, and those less than $1 \mathrm{~cm}$ are microadenomas. Examples of both are shown in - Figs. 9 and 10. With excessive growth, macroadenomas can compress surrounding structures. The most common neurological deficit noted by patients is diminished vision in the temporal fields (bitemporal hemianopsia shown in - Fig. 11) which occurs due to compression of the optic chiasm. ${ }^{18,19}$ Viewing the images and anatomy helps clarify how a large tumor could grow up and compress the closely located optic chiasm. Expansion of pituitary lesions can also lead to headaches, cranial nerve palsies (III, IV, VI, VII), cavernous sinus syndrome, and pituitary apoplexy, all of which directly result from the pituitary's anatomical proximity to structures (see - Fig. 12) that, when compressed, give rise to these symptoms. In a similar manner, lesions in structures located in proximity to the pituitary such as the hypothalamus and third ventricle can also result in loss of normal pituitary function leading to symptoms like amenorrhea, due to interference with GnRH transport to the anterior pituitary gland. The anatomic relationship explains the need for a cranial MRI in cases of hypothalamic amenorrhea, of unknown cause.

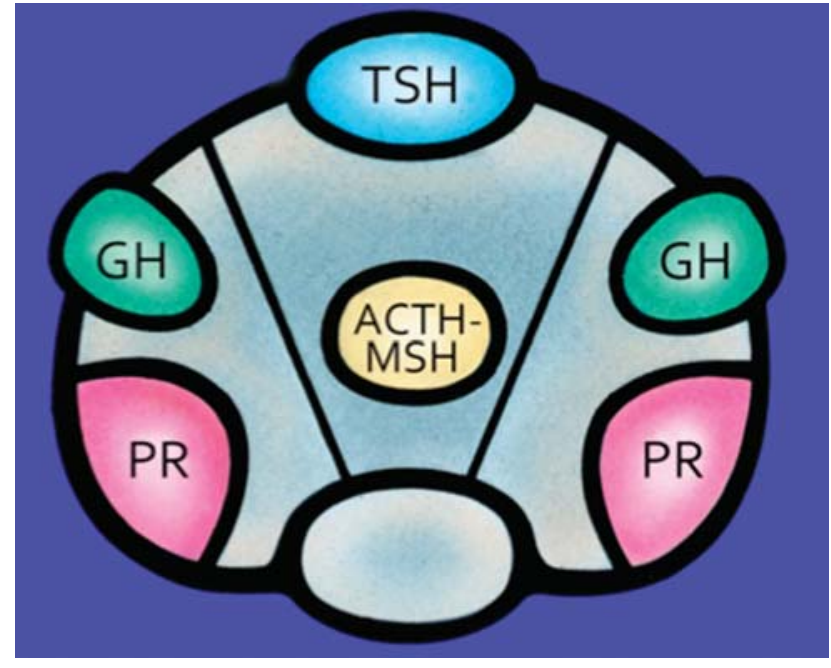

Fig. 8 Most common functional pituitary adenoma sites.

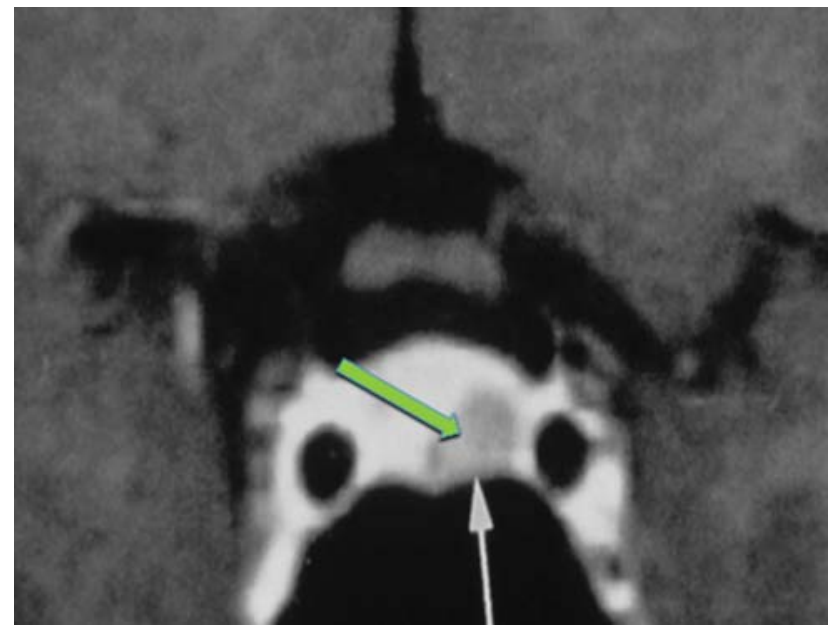

Fig. 9 A microadenoma.

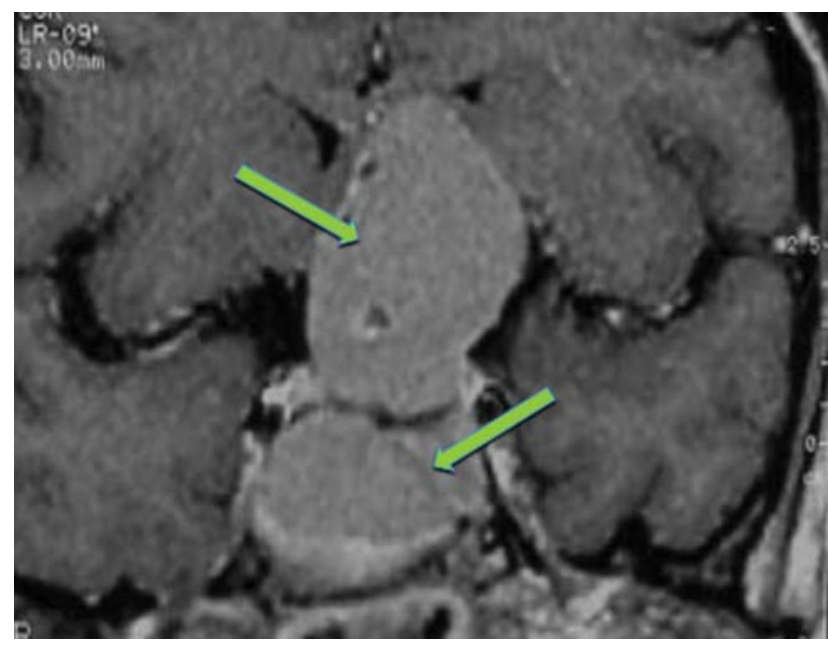

Fig. 10 A fuctional macroadenoma. 


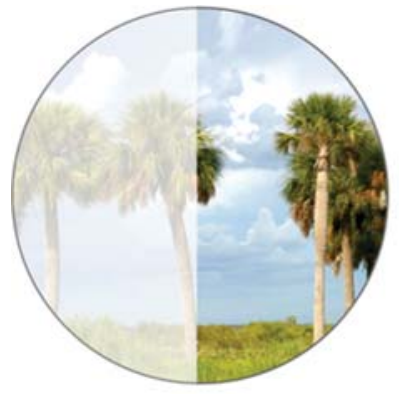

Visual Field of Left Eye

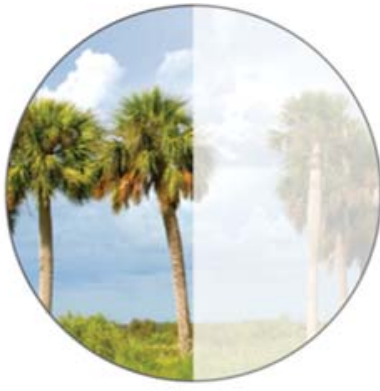

Visual Field of Right Eye
Fig. 11 Visual defect seen in bitemporal hemianopsia.

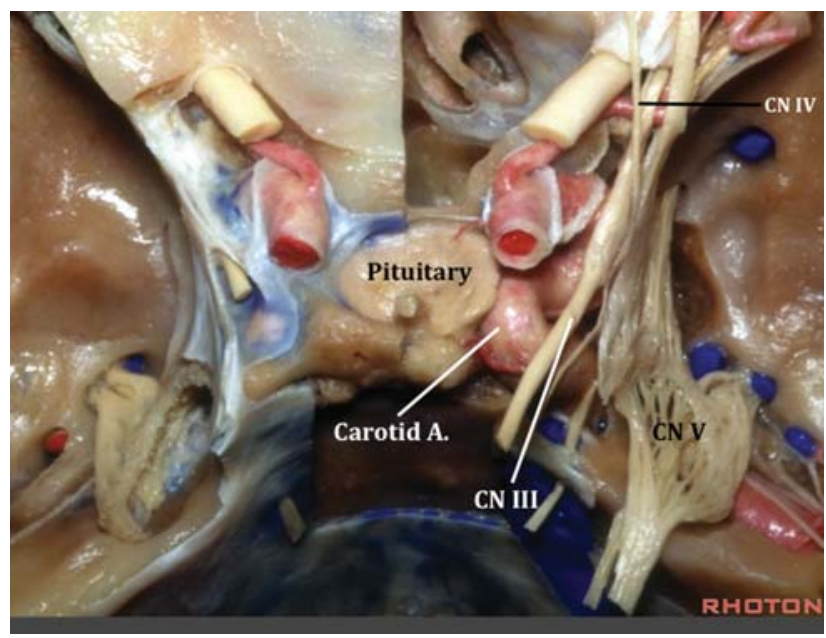

Fig. 12 Coronal view demonstrating the proximity of the pituitary with cranial nerves (CN) III, IV, and V.

\section{Treatment of Pituitary Adenomas}

After pituitary tumors are diagnosed, either medical management or surgery is usually necessary depending on the patient symptoms and tumor type. Prolactinomas are typically treated medically with dopamine agonists, less commonly with transsphenoidal surgical resection, and rarely with radiotherapy when the tumor is resistant to both surgery and dopamine agonists. ${ }^{11,12,20}$ Two dopamine agonists are available in the United States to treat prolactinomas: bromocriptine and cabergoline. Bromocriptine was the first medical treatment for prolactinomas available in the United States and is dosed daily. Common side effects include nausea, vomiting, dizziness, and postural hypotension. Cabergoline is generally better tolerated by patients and is longer acting, only needing to be dosed twice weekly. Cabergoline has been found to be more efficacious than bromocriptine, resulting in normoprolactinemia in $83 \%$ of those treated. ${ }^{11,12,20}$ Both medications are FDA Class B for pregnant women and considered safe in early pregnancy. ${ }^{11}$ As microprolactinomas rarely increase in size, women with amenorrhea due to a microadenomas who do not desire pregnancy can be treated with oral contraceptive pills (OCPs) rather than dopamine agonists. ${ }^{11}$
Indications for surgery include no response to medical management, intolerance of medical management, cerebrospinal fluid fistulas or leaks, rapid vision loss, and severe neurological deficits. ${ }^{11,12,20}$ Remission rates after surgery are generally good but have a wide reported variance, between 50 and $93 \%$ for microprolactinomas and 30 and $80 \%$ for macroprolactinomas. ${ }^{12,20}$ All other symptomatic tumor types are usually treated with surgery. However, access to the pituitary is limited by its location. Vital structures at the superior border (optic chiasm and circle of Willis), lateral borders (carotid arteries and cranial nerves), and posterior border (brainstem and basilar artery) limit surgical access to the gland. Surgically, the pituitary can be approached anteriorly, between the frontal lobe and the floor of the anterior cranial fossa, or more commonly from below through the nasal cavity and sphenoid sinus (-Fig. 13).

\section{Case Example}

A 29-year-old P0 female presented to her obstetrician-gynecologist with a 6-month history of galactorrhea and secondary amenorrhea. A prolactin level was drawn and found to be elevated at $259 \mathrm{ng} / \mathrm{mL}$, and she was referred to a reproductive endocrinologist. An MRI is scheduled and she presents to the reproductive endocrinology office prior to imaging. A thorough history and physical exam is obtained, taking care to ask about reproductive history (as pregnancy and breast feeding are the most common etiology of elevated prolactin), current medications (specifically verapamil, neuroleptics, antipsychotics, and antidepressants which can elevate prolactin levels), estrogen use, chronic stress, history of head trauma, history or symptoms of hypothyroidism, and chronic hepatic or renal disease. Screening for significant history is negative.

Labs ordered include a repeat prolactin level, TSH, basic metabolic panel (BMP), and pregnancy test. Repeat prolactin is $302 \mathrm{ng} / \mathrm{mL}$, and all other testing returns within normal range. The pituitary MRI is completed, consistent with a 9-mm pituitary adenoma. The patient is started on $0.25 \mathrm{mg}$ of

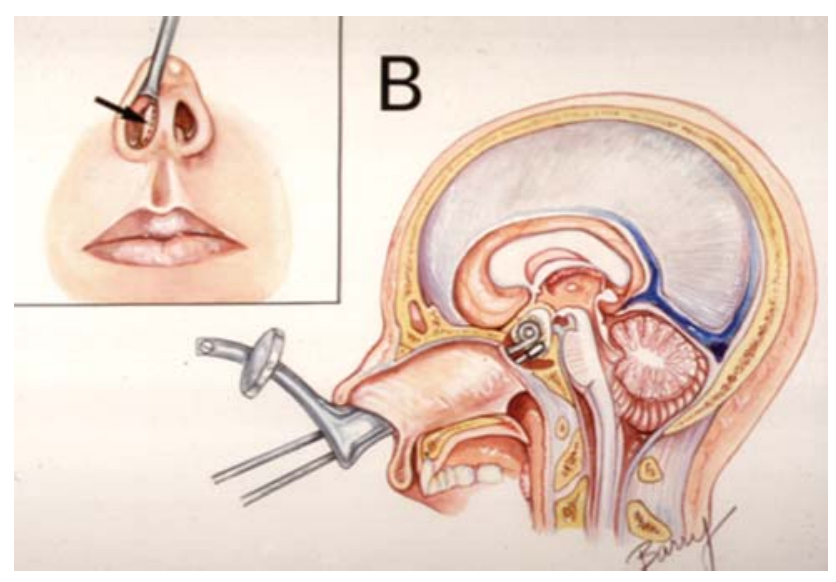

Fig. 13 Transsphenoidal approach to pituitary tumors. Image drawn by medical illustrator Margaret E. Barry, from the Rhoton neuroanatomy laboratory. (Reprinted with permission from Rhoton AL. The stellar region. Neurosurgery 2002;53:364.) 
cabergoline twice weekly, and asked to return to clinic in 1 month.

She returns 1 month later and reports persistence of her symptoms. In addition, she now reports difficultly seeing in her peripheral visual fields. A repeat prolactin level is drawn and found to be $398 \mathrm{ng} / \mathrm{mL}$. The dose of cabergoline is increased to $0.5 \mathrm{mg}$ twice weekly. The patient calls up the office later the next week to report severe nausea and dizziness with the increased dose, asking for other options. Bromocriptine is tried but her nausea and vomiting are worse. Given the worsening symptoms and inability to tolerate medical management, a referral is placed to neurosurgery. The patient undergoes transsphenoidal resection of the microprolactinoma 6 weeks later with resolution of her symptoms.

\section{Conclusion}

The critical parts of the female reproductive system are the hypothalamus, pituitary, and female genital tract with the ovaries clinically and endocrinologically linked to the brain and reproduction. Important scientific discoveries made in the past 100 years have allowed the growth of the field of endocrinology, as we now have the knowledge and means to manipulate the hypothalamic-pituitary-gonadal axis. This project serves to demonstrate that any disease process that interrupts pituitary or hypothalamic function will alter reproductive events and can lead to other neurological deficits. Through this work with anatomical dissections and their photographic documentation, we aim to reinforce how pathological pituitary function leads to altered reproductive processes and highlight the neuroanatomy and neurohormonal manipulation underlying the care provided by reproductive endocrinologists.

\section{Acknowledgment}

We dedicate the work to the memory of Albert L. Rhoton Jr, who loved the brain and neuroanatomy. He inspired all contributing authors, to follow their field of study based on his awe and dedication to the study of the brain, pituitary gland, and medicine.

\section{References}

1 Lunenfeld B. Historical perspectives in gonadotrophin therapy. Hum Reprod Update 2004;10(06):453-467

2 Sellar Region | Neurosurgery | Oxford Academic. Available at: https:// academic.oup.com/neurosurgery/article/51/suppl_4/S1-335/2728103. Accessed August 30, 2018
3 Larkin S, Ansorge O. Development and microscopic anatomy of the pituitary gland. Endotext; 2000. Available at: MDText.com, Inc.

4 Asa SL, Ezzat S. The pathogenesis of pituitary tumors. Annu Rev Pathol 2009;4:97-126

5 Ostrom QT, Gittleman H, Farah P, et al. CBTRUS statistical report: primary brain and central nervous system tumors diagnosed in the United States in 2006-2010. Neuro-oncol 2013;15(Suppl 2): ii1-ii56

6 Ostrom QT, Gittleman H, Liao P, et al. CBTRUS Statistical Report: primary brain and other central nervous system tumors diagnosed in the United States in 2010-2014. Neuro-oncol 2017;19 (Suppl 5):v1-v88

7 Nammour GM, Ybarra J, Naheedy MH, Romeo JH, Aron DC. Incidental pituitary macroadenoma: a population-based study. Am J Med Sci 1997;314(05):287-291

8 Chambers EF, Turski PA, LaMasters D, Newton TH. Regions of low density in the contrast-enhanced pituitary gland: normal and pathologic processes. Radiology 1982;144(01):109-113

9 Ezzat S, Asa SL, Couldwell WT, et al. The prevalence of pituitary adenomas: a systematic review. Cancer 2004;101 (03):613-619

10 Hall WA, Luciano MG, Doppman JL, Patronas NJ, Oldfield EH. Pituitary magnetic resonance imaging in normal human volunteers: occult adenomas in the general population. Ann Intern Med 1994;120(10):817-820

11 Molitch ME. Diagnosis and treatment of pituitary adenomas: a review. JAMA 2017;317(05):516-524

12 Primeau V, Raftopoulos C, Maiter D. Outcomes of transsphenoidal surgery in prolactinomas: improvement of hormonal control in dopamine agonist-resistant patients. Eur J Endocrinol 2012;166 (05):779-786

13 Inoshita N, Nishioka $\mathrm{H}$. The 2017 WHO classification of pituitary adenoma: overview and comments. Brain Tumor Pathol 2018;35 (02):51-56

14 Skugor M, Hamrahian AH. Pituitary disorders. In: Carey WD, ed. Current Clinical Medicine. 2nd ed. Philadelphia, PA: Saunders/ Elsevier; 2010;408-415.

15 Ben-Shlomo A, Melmed S. Acromegaly. Endocrinol Metab Clin North Am 2008;37(01):101-122, viii

16 Brue T, Castinetti F. The risks of overlooking the diagnosis of secreting pituitary adenomas. Orphanet J Rare Dis 2016;11(01): 135

17 Cote DJ, Smith TR, Sandler CN, et al. Functional gonadotroph adenomas: case series and report of literature. Neurosurgery 2016;79(06):823-831

18 Monson JP. The epidemiology of endocrine tumours. Endocr Relat Cancer 2000;7(01):29-36

19 Losa M, Mortini P, Barzaghi R, Franzin A, Giovanelli M. Endocrine inactive and gonadotroph adenomas: diagnosis and management. J Neurooncol 2001;54(02):167-177

20 Kreutzer J, Buslei R, Wallaschofski H, et al. Operative treatment of prolactinomas: indications and results in a current consecutive series of 212 patients. Eur J Endocrinol 2008;158(01):11-18 